\title{
CB Research Square \\ Climate Suitable Conditions Index and Autochthonous Dengue Infections: an Early Warning Index
}

\author{
Xinting Lu \\ Queensland University of Technology \\ Hilary Bambrick \\ Queensland University of Technology \\ Francesca D. Frentiu \\ Queensland University of Technology

\section{Xiaodong Huang} \\ Queensland University of Technology

\section{Callan Davis} \\ Queensland University of Technology
}

\section{Zhongjie Li}

Chinese Center for Disease Control and Prevention

\section{Weizhong Yang}

Chinese Academy of Medical Sciences \& Peking Union Medical College

Gregor J. Devine

QIMR: QIMR Berghofer Medical Research Institute

Wenbiao Hu ( $\nabla$ w2.hu@qut.edu.au )

Queensland University of Technology https://orcid.org/0000-0001-6422-9240

\section{Research Article}

Keywords: Dengue transmission, Suitable conditional index, Aedes. aegypti, Ae. albopictus, Interactive effect

Posted Date: December 28th, 2021

DOI: https://doi.org/10.21203/rs.3.rs-1165147/v1

License: (0) (1) This work is licensed under a Creative Commons Attribution 4.0 International License. Read Full License 


\section{Abstract}

Background: Aedes aegypti is the primary vector of dengue and contributes to most major epidemics of this virus worldwide. Aedes albopictus is also blamed for certain epidemics, as the 2014 Guangdong dengue outbreak. In Guangdong province, Ae. albopictus is thought to be the dominant vector species, with Ae. aegypti absent from most areas. Whether or not primary mosquito vectors are present, optimal climatic conditions for dengue vector species may play a substantial role in epidemics of the virus. We hypothesise that although vector species are required to initiate and establish an outbreak, favourable weather conditions may then grow and perpetuate the outbreak via complex effects on vector sympatry or interactions.

Methods: Vector spices-specific suitable conditions index $(\mathrm{SCl})$ and autochthonous dengue case data were fitted to negative binomial (NB) regression models. After accounting for potential confounders, we assessed the relationship between $\mathrm{SCl}$ and autochthonous dengue cases. We assumed SCl interaction was a proxy for vector species sympatry and SCl difference a proxy for interspecies competition. Finally, we explored the relationship between these assumed conditions and the autochthonous dengue case.

Results: Autochthonous dengue cases are associated negatively with $\mathrm{SCl}$ for Ae.albopictus and positively with $\mathrm{SCl}$ for Ae.aegypti. According to the NB regression models, autochthonous dengue cases increased 4\% (Incident Rate Ratio (IRR): 1.04, 95\% Cl: 1.02, 1.06) for every unit increase in SCl for Ae. aegypti, but decreased by 3\% (IRR: 0.97, 95\% Cl: 0.96, 0.99) for Ae. albopictus SCl. There was also an interaction between two SCls and a positive effect of the difference in SCls on autochthonous dengue cases. These findings support the hypothesis that vector sympatry and interactions may influence the risk of a dengue outbreak.

Conclusions: Our results confirm the hypothesis that the dengue virus is more transmissible in regions with warmer weather conditions (high SCl for Ae. aegypti). SCl of Ae. aegypti would be a valuable index to predict dengue transmission even in the absence of Ae. aegypti but with Ae. albopictus present. The results also support that the $\mathrm{SCl}$ is beneficial for evaluating dengue outbreak risk in terms of vector sympatry and interactions in the absence of entomology data in future research.

\section{Background}

Dengue has risen rapidly throughout the world in recent decades. According to model estimates, 390 million dengue virus infections occur yearly [1], and 3.9 billion people are at risk of infection [2]. Dengue cases reported to World Health Organization (WHO) have climbed from 505,430 in 2000 to over 2.4 million in 2010 and 5.2 million in 2019 [3]. To date, dengue is the most prevalent human arboviral infection worldwide. Dengue is an arthropod-borne virus (arbovirus) infection caused by four virus serotypes, transmitted by the two Aedes mosquito species: Ae. aegypti and Ae. albopictus [4]. Ae. aegypti and Ae. albopictus, once zoophilic forest species from sub-Saharan Africa and Asia respectively, are now widespread globally due to rapid human population growth and international trade in the last century and climate change more recently $[5,6]$.

Ae. aegypti is considered highly urban-adapted, while Ae. albopictus can thrive in peridomestic and rural environments over a wide range of tropical, subtropical and temperate climates [7]. In China, Ae. aegypti was once only present in the areas of the tropical zone below $22^{\circ} \mathrm{N}$, such as Hainan Island, Leizhou peninsula in Guangdong province and coastal area in Beibu Gulf of Guangxi province. However, since the 2000s, Ae. aegypti has also been found in some bordering counties in Yunnan province, located between $22^{\circ} \mathrm{N}$ and $25^{\circ} \mathrm{N}$ [8]. In contrast, Ae. albopictus has a wide distribution in both tropical and temperate regions of China from $41^{\circ} \mathrm{N}$ to the country's southern reaches [9]. Based on historical climatic data from the last decade, most areas where Ae. albopictus became established had an annual mean temperature above $11^{\circ} \mathrm{C}$ and annual mean precipitation above $500 \mathrm{~mm}$ [10].

Ae. aegypti is considered the principal dengue vector and has contributed to a global resurgence of dengue epidemics in the past three decades. All major epidemics of dengue have occurred only in areas where Ae. aegypti was found [11]. Conversely, Ae. albopictus is considered to play a relatively minor role in dengue virus transmission compared to Ae. aegypti, and is not considered an 'efficient' epidemic vector [11, 12]. In China, dengue outbreaks in Hainan province in 1980 and 1985-86 and Xishuangbanna of Yunnan province in 2013 were typically attributed to Ae. aegypti $[13,14]$. However, outbreaks, largely of classical dengue (i.e., few cases of severe disease) in Guangdong, Fujian, and Zhejiang province between 2004 and 2010, were

Page 2/14 
caused solely by Ae. albopictus in the absence of Ae. aegypti $[15,16]$. In 2014, China's largest dengue outbreak to date (or at least since dengue became a notifiable disease in 1989) occurred in Guangdong, where Ae. albopictus appears to be the sole dengue vector [17].

The dynamics of dengue transmission and outbreak are complicated, including hosts (humans), pathogens and mosquito vectors with their ecological interactions. The WHO recommends that many dengue-endemic nations use vector surveillance to provide a quantitative evaluation of dengue vector population fluctuations in the number and geographical distribution to forecast outbreaks and assess management options [18]. However, dengue transmission usually depends on a range of factors, including serostatus of the human host population, mosquito density and climate, rather than at a fixed entomologic threshold [19]. Additionally, there has been minimal evidence of meaningful correlations between vector biomass indices and dengue transmission, which might be used to forecast epidemics consistently [20]. Furthermore, the geographical variability in the association between dengue vector abundance and transmission or outbreak occurrence is poorly understood, suggesting that locality-specific vector population indices may be critical in forecasting dengue transmission increases [21], and other data may have better potential as predictors.

Climate is a crucial environmental determinant of vector geographical distribution and vectorial capacity. Climate factors such as temperature and precipitation and their seasonal patterns can fundamentally affect mosquito population dynamics and individual features relevant to vector biologies like development, reproduction and survival, and consequently dengue virus transmission patterns [22-24]. A sufficiently warm ambient temperature is also critical for virus replication and dissemination to the salivary glands in female mosquitoes. Conversely, cooler temperatures slow this viral amplification, and if the development time of the pathogen exceeds the life span of the infected mosquito, transmission cannot occur [7, 25]. Previous studies have estimated suitable temperatures that support dengue transmission [26-29]. Mordecai et al. have synthesised the existing empirical evidence on temperature suitability for vector and pathogen survival and used a mechanistic approach to ascertain suitable temperatures for vector capacity in dengue transmission [30]. Based on these identified suitable temperature ranges for dengue vectorial capacity, we have previously developed the Suitable Conditions Index (SCl) for Ae. aegypti and Ae. albopictus to define their potential vector capacity. SCI ranks geographic locations based on their climatic suitability for each vector species that transmits dengue and theoretically defines the spatial parameters for transmission [31]. By incorporating SCl into an outbreak epidemiologic study, it is possible to investigate the potential value of $\mathrm{SCl}$ data for dengue transmission or outbreak prediction. Here, we use the case of the Guangdong dengue outbreak in 2014 to assess the potential of a climate-based SCl as a local dengue outbreak early warning index.

\section{Methods}

\section{Study area}

Guangdong (at $109^{\circ} 39^{\prime} 53^{\prime \prime} \mathrm{E}$ to $117^{\circ} 18^{\prime} 51^{\prime \prime} \mathrm{E}$ and $20^{\circ} 13^{\prime} 24^{\prime \prime} \mathrm{N}$ to $\left.25^{\circ} 31^{\prime} 10^{\prime \prime}\right)$ province is in southern China, with approximately $179,800 \mathrm{~km}^{2}$ in area. Guangdong is the most populous province in China, with 126.01 million inhabitants (7th National Population Census), accounting for $8.93 \%$ of Mainland China's population [32]. Guangdong has a humid subtropical climate; winters are short, mild, and relatively dry, while summers are long, hot, very wet and influenced by the Asian monsoon season [33].

\section{Data collection}

Data on locally acquired and imported dengue cases reported from Guangdong in 2014 were collected from the National Notifiable Infectious Diseases Reporting Information System. Dengue has been a statutorily notifiable communicable disease in China since 1989 [34]. Dengue is diagnosed according to the national surveillance protocol with standardised case definitions, including clinically diagnosed and laboratory-confirmed dengue cases. An imported case was defined as one for which the patient had travelled abroad to a dengue-endemic country within 15 days of the onset of illness. In some cases, importation was defined based on laboratory results showing that the infection dengue virus had a sequence similarity in the premembrane (prM) and envelop (E) glycoproteins as (prM-E) region of the virus genome compared with viruses isolated from the putative source region where the patient had travelled [35]. In the absence of meeting the criteria for an imported case, a dengue case was considered an autochthonous dengue case. 
$\mathrm{SCl}$ has been developed in our previous research [31] to define climate suitability for each species of Ae. aegypti and Ae. albopictus. Based on the optimal temperature range for vectorial capacity in the transmission identified by Mordecai et al. using robust mechanistic models [30], the SCl was defined as the product of the number of suitable months (where the monthly minimum and maximum temperature is within the determined temperature range) and the average monthly precipitation (as a weight). The input data for the SCl model were available on an annual interval and at the county scale. Therefore, we produced $\mathrm{SCl}$ values for each species of Ae. aegypti and Ae. albopictus in 2014 for 124 counties/districts in Guangdong.

Normalised Difference Vegetation Index (NDVI) data were extracted from NASA's Moderate Resolution Imaging Spectroradiometer (MODIS) website (MODIS Web (nasa.gov)). NDVI data are used to characterise and monitor the global distribution of vegetation conditions and are often used to model global and regional climate [36]. We utilised MODIS Vegetation Indices (MOD13A3 product) version 6 data provided monthly at the 1-kilometre spatial resolution, then aggregated annually and at the county scale [37]. In addition, human population data were obtained from the Sixth National Population Census data (2010), which is available from the National Bureau of Statistics of China (http://www.stats.gov.cn/enGliSH/).

\section{Negative binomial regression (NB) models}

We fitted a fixed-effects NB model to assess the relationship between autochthonous dengue cases and potential predictors, including SCl for each vector species, NDVI, human population density, and the number of imported dengue cases. An NB model was used to account for the over-dispersed count of the dengue case (Table 1). A log-scale population was used as an offset to control for variation in human population size. Univariable and multivariable analyses were implemented successively. In univariable analyses, we consider each potential predictor in an NB regression model to identify significant predictors. In a multivariable analysis, we examined the potential effect of significant predictors on dengue case occurrence (presented as incident rate ratio, IRR). Model goodness of fit was assessed by Bayesian information criterion (BIC), where smaller values of BIC indicate a better fit.

Table 1

Summary statistics for autochthonous dengue cases, imported dengue cases, Normalized Difference Vegetation Index (NDVI), human population density, SCl for Ae. aegypti and SCI for Ae. albopictus in 124 counties/districts of Guangdong, 2014

\begin{tabular}{|llllll|}
\hline Variable & Mean & SD & Median & Minimum & Maximum \\
\hline Autochthonous dengue & 361.79 & 1387.31 & 10.50 & 0 & 11837 \\
\hline Imported dengue & 0.62 & 2.08 & 0 & 0 & 15 \\
\hline NDVI & 185.40 & 28.42 & 193.22 & 94.78 & 255.00 \\
\hline Population density & 2131.33 & 4008.42 & 502.35 & 76.91 & 21686.58 \\
\hline SCl-Ae. aegypti & 102.48 & 22.84 & 103.79 & 45.42 & 154.70 \\
\hline SCl-Ae. albopictus & 71.79 & 20.69 & 63.60 & 34.92 & 138.68 \\
\hline SD: Standard deviation SCl: Suitable Conditions Index NDVI: Normalized Difference Vegetation Index \\
\hline
\end{tabular}

We also examined the potential interaction between $\mathrm{SCl}$ for Ae. albopictus and SCl for Ae. aegypti (SCl-Ae.aegypti $\times \mathrm{SCl}-A e$. albopictus). We assumed the $\mathrm{SCl}$ was a proxy for vector density; a high level of SCl represented high vector density and vice versa. The interaction between the two vectors was examined by assessing how the effect of the $\mathrm{SCl}$ of a specific vector on autochthonous dengue case occurrence changed when the $\mathrm{SCl}$ of another vector changed. The SCl difference (defined as SClAe.aegypti-SCl-Ae. albopictus) was also fitted to the NB model. As Ae. albopictus has a different niche to Ae. aegypti in terms of feeding preferences and, critically for the $\mathrm{SCl}$, thermal range, it was reasonable to assume that more considerable difference may indicate a reduced probability of ecological constraints between the two mosquito species. We use the SCI difference to explore the correlation between the probability of ecological constraints and dengue transmission risk. The regression analysis was implemented in Stata/SE 16.0 (StataCorp, TX, USA) using "xtnbreg" (Fixed-effects, random-effects, \& population-averaged negative binomial models) [38]. 


\section{Results}

\section{Descriptive analyses}

Summary statistics for autochthonous dengue cases, imported cases, NDVI, human population density, SCl for Ae. aegypti and SCI for Ae. albopictus are presented in Table 1. In 2014, there were 44,939 (44,862 autochthonous and 77 imported) dengue cases recorded from 107 out of 124 counties in Guangdong, number of cases per county/district ranged from 0 to 11,846 (mean number of cases per county/district: 362.41 , standard deviation: 1,388.57). The mean annual dengue incidence averaged 28.9 cases per 100,00 population, with the highest incidence $(532.6 / 100,000)$ being reported from Baiyun District, followed by Liwan (497.3/100,000), Yuexiu (414.1/100,000), and Haizhu District (385.2/100,000), all of which are located in Guangzhou, the capital city of Guangdong (Fig. 1a).

Guangdong has the most suitable climatic conditions for dengue transmission for each vector (Fig. 1b, c). SCl for Ae. aegypti was highest in the Leizhou peninsula below $22^{\circ} \mathrm{N}$ and western coastal areas between $22-23^{\circ} \mathrm{N}$, with gradually reducing suitability moving further north, and the lowest was observed in the furthest northern part (Fig. 1b). SCl for Ae. albopictus was less likely to be distributed evenly through low to high latitude (Fig. 1c). Regions with the highest SCI for both vectors overlapped and located in the western coastal areas below $23^{\circ} \mathrm{N}$ (Fig. 1b,c).

The relationship between autochthonous dengue incidence, latitude, imported case and SCl for the two vector species is presented in Fig. 2. The correlation pattern between autochthonous dengue case and latitude was consistent between the two vector species; incidence and $\mathrm{SCl}$ were high in $23^{\circ} \mathrm{N}$ (Fig. 2a and b). The relationship between imported dengue cases, vectors and autochthonous dengue cases indicated a strong relationship between autochthonous and imported dengue cases, which may remain independent over various levels of $\mathrm{SCl}$ for each vector species (Fig. $2 \mathrm{c}$ and d).

The relationship between autochthonous dengue incidence and $\mathrm{SCl}$ for Ae. aegypti was examined when $\mathrm{SCl}$ for Ae. albopictus was held constant at four levels from low to high (34.9 to $58.1,58.2$ to $63.6,63.8$ to 84.5 and 85.5 to 141.8 ), with results presented in Fig. 2e. There was a moderate relationship between dengue incidence and SCl for Ae. aegypti when $\mathrm{SCl}$ of $A e$. albopictus was held constant at 34.9 to 58.1 (e.g., minimum to mean - one SD) (Fig. 2e).

\section{Spearman correlation}

Spearman correlation coefficients between any two variables considered in this study are presented in Table 2 . There was a moderate relationship between autochthonous dengue incidence and SCl for Ae. aegypti $\left(\mathrm{r}_{\mathrm{s}}=0.41, p=0.000\right)$, while there was a weak correlation between incidence and SCI for Ae. albopictus $\left(r_{s}=-0.18, p=0.000\right)$. There was a moderate correlation between dengue incidence and difference in $\mathrm{SCl}\left(\mathrm{r}_{\mathrm{S}}=0.53, p=0.000\right)$. There was a moderate correlation between autochthonous dengue incidence and the other variables, including imported cases, NDVI and human population density. There was a moderate relationship between the two SCls $\left(\left(r_{s}=0.43, p=0.000\right)\right.$. 
Table 2

Correlation between autochthonous dengue annual incidence and exploratory variables

\begin{tabular}{|c|c|c|c|c|c|c|c|}
\hline & $\begin{array}{l}\text { Autochthonous } \\
\text { dengue }\end{array}$ & Sci-Ae.aegypti & Sci-Ae.albopictus & $\begin{array}{l}\text { Imported } \\
\text { dengue }\end{array}$ & NDVI & $\begin{array}{l}\text { Population } \\
\text { density }\end{array}$ & $\begin{array}{l}\text { Difference } \\
\text { in SCl }\end{array}$ \\
\hline $\begin{array}{l}\text { Autochthonous } \\
\text { dengue }\end{array}$ & 1.00 & & & & & & \\
\hline \multirow[t]{2}{*}{ Sci-Ae.aegypti } & 0.41 & 1.00 & & & & & \\
\hline & $(0.000)$ & & & & & & \\
\hline \multirow[t]{2}{*}{ Sci-Ae.albopictus } & -0.18 & 0.43 & 1.00 & & & & \\
\hline & $(0.049)$ & $(0.000)$ & & & & & \\
\hline \multirow[t]{2}{*}{ Imported dengue } & 0.50 & 0.18 & -0.20 & 1.00 & & & \\
\hline & $(0.000)$ & $(0.048)$ & $(0.024)$ & & & & \\
\hline \multirow[t]{2}{*}{ NDVI } & -0.56 & -0.24 & 0.34 & -0.42 & 1.00 & & \\
\hline & $(0.000)$ & $(0.007)$ & $(0.000)$ & $(0.000)$ & & & \\
\hline \multirow{2}{*}{$\begin{array}{l}\text { Population } \\
\text { density }\end{array}$} & 0.64 & 0.34 & -0.18 & 0.53 & -0.73 & 1.00 & \\
\hline & $(0.0000)$ & $(0.000)$ & $(0.044)$ & $(0.000)$ & $(0.000)$ & & \\
\hline \multirow[t]{2}{*}{ Difference in $\mathrm{SCl}$} & 0.53 & 0.53 & -0.46 & 0.32 & -0.51 & 0.46 & 1.00 \\
\hline & $(0.000)$ & $(0.00)$ & $(0.000)$ & $(0.000)$ & $(0.00)$ & $(0.000)$ & \\
\hline
\end{tabular}

\section{NB Regression models}

Results of univariable analyses (Model I in Table 3) indicated that SCl for Ae. aegypti, the difference between SCl for Ae. aegypti and $\mathrm{SCl}$ for Ae. aegypti (Difference in SCl), NDVI, and imported case were significant predictors of dengue transmission. Results of multivariable models without the interact terms (Model II and III) indicated that autochthonous dengue cases increased $4 \%$ (IRR: 1.04, 95\% Cl: 1.02, 1.06) for one unit increase in SCl for Ae. aegypti, but decreased by 3\% (IRR: 0.97, 95\% Cl: 0.96, 0.99) for every unit increase in SCl for Ae. albopictus. Autochthonous dengue cases increased 28\% (IRR: 1.28, 95\% Cl: 1.02, 1.60) and 4\% (IRR: $1.04,95 \% \mathrm{Cl}: 1.02,1.06)$ for one unit increase in imported case and difference in SCl, respectively. Dengue case decreased $3 \%$ (IRR: $0.97,95 \% \mathrm{Cl}: 0.96,0.99)$ for one unit increase in NDVI. A significant interaction effect between SCl of Ae. albopictus and $\mathrm{SCl}$ of $A$ e. aegypti was found, where $\mathrm{SCl}$ of Ae. albopictus significantly reduced the effect $\mathrm{SCl}$ of $A$ e. aegyption autochthonous dengue cases (Table 3 Model IV). To avoid possible collinearity between NDVI and population density $\left(r_{s}=-0.73, p=0.000\right.$, Table 2), more importantly, the population density was not a significant predictor in multivariable models), the population density was excluded from NB regression analyses. 
Table 3

Incident rate ratio (IRR) and 95\% confidence interval (Cl) for potential predictors of autochthonous dengue case in Guangdong, 2014

\begin{tabular}{|c|c|c|c|c|}
\hline \multirow[t]{2}{*}{ Variable } & Model $l^{\mathrm{a}}$ & Model II ${ }^{b}$ & Model IIIC & Model IV ${ }^{d}$ \\
\hline & IRR (95\% Cl) & IRR (95\% Cl) & IRR (95\% Cl) & IRR $(95 \% \mathrm{Cl})$ \\
\hline $\mathrm{SCl}-A e$. aegypti & $1.03(1.02,1.05)$ & $1.04(1.02,1.06)$ & - & $1.06(1.03,1.09)$ \\
\hline SCl-Ae. albopictus & $0.99(0.97,1.01)$ & $0.97(0.95,0.99)$ & - & - \\
\hline Difference in SCl & $1.05(1.04,1.07)$ & - & $1.04(1.02,1.06)$ & - \\
\hline NDVI & $0.95(0.94,0.97)$ & $0.98(0.96,0.99)$ & $0.98(0.96,0.99)$ & $0.97(0.96,0.99)$ \\
\hline Imported case & $1.59(1.31,1.93)$ & $1.28(1.02,1.61)$ & $1.28(1.02,1.60)$ & $1.28(1.01,1.61)$ \\
\hline SCl-Ae.aegypti × SCl-Ae. albopictus & - & - & - & $0.99(0.99,0.99)^{\mathrm{e}}$ \\
\hline BIC & & 1165.30 & 1162.67 & 1165.91 \\
\hline \multicolumn{5}{|l|}{ a Univariable models } \\
\hline \multicolumn{5}{|c|}{${ }^{\mathrm{b}}$ Multivariable models with predictors being SCl-Ae aegypti, NDVI, Imported case } \\
\hline \multicolumn{5}{|c|}{${ }^{\mathrm{c}}$ Multivariable models with predictors being Difference in SCI, NDVI, Imported case } \\
\hline \multicolumn{5}{|c|}{${ }^{\mathrm{d}}$ Multivariable model III with interact term SCl-Ae.aegypti $\times \mathrm{SCl}$-Ae. albopictus } \\
\hline \multicolumn{5}{|l|}{ e $0.9998(0.9996,0.9999), p=0.001$} \\
\hline \multicolumn{5}{|l|}{ SCl: Suitable condition index } \\
\hline \multicolumn{5}{|c|}{ NDVI: Normalized Difference Vegetation Index } \\
\hline BIC: Bayesian information criterion & & & & \\
\hline
\end{tabular}

\section{Discussion}

We investigated the association between $\mathrm{SCl}$ and autochthonous dengue cases in this study and the value of $\mathrm{SCl}$ as a predictor of dengue outbreak risk. We found SCl was associated with autochthonous dengue cases, implying SCl might be beneficial in forecasting the probability of local dengue transmission or outbreak occurrence. We also discovered a statistically significant interaction between $\mathrm{SCl}$ for Ae. albopictus and $\mathrm{SCl}$ for Ae. aegypti, which invites the hypothesis that vector species interactions may also influence dengue virus transmission.

We found opposing relationships between species-specific SCl and autochthonous dengue occurrence in Guangdong: $\mathrm{SCl}$ for $A e$. aegypti was positively associated with disease cases while $\mathrm{SCl}$ for Ae. albopictus was negatively related to local cases. This finding is not surprising because there are still several significant gaps in understanding correlations between climate suitability and virus transmission probability and intensity. Although temperature-dependent transmission models prove valuable for predicting vector spread and dengue transmission, most of these modelling studies find a very broad correlation of transmission occurring within a temperature range $[22,39]$. Few can sufficiently capture transmission dynamics to provide reliable predictions. Human mobility and behaviour, urban development and microclimates, aquatic habitat supply, vector control techniques may all impact dengue transmission [23, 40-45]. These characteristics may be as important as climate suitability in determining dengue transmission risk. Moreover, transmission dynamics and bionomic responses differ across vector species, mosquito populations and dengue virus serotypes [20,46,47]. While some potential confounders have been adjusted in our study, other elements may contribute to dengue transmission that we have not considered. Finally, whether primary mosquito vectors are present, optimal climatic conditions for dengue vector species may play a substantial role in the outbreak. This suggests the possibility that vector 
species are a prerequisite for initiating and establishing a dengue outbreak; suitable conditions may expand and maintain the outbreak through complicated effects on vector sympatry or interactions.

Although SCl was not equivalent to mosquito species absence/presence or densities revealed only by entomological surveillance data, specific vector mosquitoes should only occur at a suitable habitat with favourable meteorological conditions. Our findings indicate the possibility that $\mathrm{SCl}$ might be indirectly associated with autochthonous dengue cases through the variation in dengue vector population, which means that $\mathrm{SCl}$ might be beneficial in dengue transmission or outbreaks prediction in the absence of entomological data. This might be meaningful in some dengue-endemic countries where vector absence/presence or density data are usually unavailable and, more importantly, there has been no reliable indication of any consistent association between entomological indices and dengue cases [20].

Our results suggested a significant interaction between $\mathrm{SCl}$ for $A$ e. aegypti (main effect) and $\mathrm{SCl}$ for Ae. albopictus, which means the expected effect of $\mathrm{SCl}$ for $A e$. aegyption autochthonic dengue cases may change over the various levels of $\mathrm{SCl}$ for $A e$. albopictus. Specifically, a one-unit increase in SCI for Ae. aegypti may indicate a higher risk of dengue in a region with a low SCI of Ae. albopictus than in a region with a high SCl of Ae. albopictus. Given that regions with high SCl for each Aedes vector have some overlaps in Guangdong, the coexistence of these two vectors is theoretically possible. In areas sympatric for the two species, declining population and displacement of Ae. aegypti have been reported as a result of Ae. albopictus superiority in resource competition at the larval stage and asymmetric sterilisation at the adult stage following interspecific mating [48]. It has also been supposed that $A$. aegypti prefers to reproduce in areas devoid of Ae. albopictus [49]. Although there is no evidence of vector competitiveness from a field study in Guangdong, recent evidence from a laboratory experiment suggests competitive displacement is theoretically possible. Larvae of the two species were mixed, allowed to emerge and then cycled through six generations, with the results that Ae. aegypti from the Leizhou peninsula were suppressed (a combination of generation time and abundance was used to judge competitiveness) by Ae. albopictus from Guangzhou but not by Ae. albopictus from other cities

[50]. This finding warrants further exploration because the sympatric habit for the two species is expected to expand to additional areas in China [51].

We also observed that the difference in $\mathrm{SCl}$ was positively associated with dengue transmission. The two Aedes species are usually distinct in terms of ecological environment, feeding preferences and, critically for the $\mathrm{SCl}$, thermal range. Therefore, high $\mathrm{SCl}$ for Ae. aegypti correlating with low SCl for Ae. albopictus is meaningful, indicating that a larger difference in $\mathrm{SCl}$ may indicate less competition between the two mosquito species. In other words, areas that are optimally suitable for both species (i.e. low difference in $\mathrm{SCl}$ ) may, somewhat counterintuitively, produce a decreased dengue transmission risk potentially through the competitive displacement of one species by the other.

There are several limitations to our study. SCl is estimated using annual data and may not accurately capture seasonal variation and time-lag effects in dengue case occurrence. A finer scale of $\mathrm{SCl}$ is thus needed in any subsequent spatiotemporal analysis in this geographic region. Since $\mathrm{SCl}$ is not equivalent to dengue vector density, $\mathrm{SCl}$ should be validated in future studies against actual vector surveillance or entomology survey data. However, as a first step, our SCl provides valuable insight into the possible relationship between optimal transmission environments and broad disease predictions. Further parameters, such as environmental variation (e.g., oviposition habitat availability, seasonal and daily temperature fluctuation) and socioeconomic variables, should be included in the $\mathrm{SCl}$ to improve prediction efficiency. Enhanced SCl may be beneficial for extrapolating the possible geographic range of transmission beyond the existing environmental context (e.g., under climate change and for newly invading pathogens).

\section{Conclusions}

This study has shown that climate-based SCl is beneficial for evaluating dengue outbreak risk and the potential effect of possible vector competition on dengue transmission. We suggest that the dengue virus in Ae. albopictus vector may be more transmissible in a region with warmer weather conditions (high $\mathrm{SCl}$ of $A$ e. aegypti). $\mathrm{SCl}$ of $A$ e. aegypti could be used to predict dengue transmission even in locations lacking Ae. aegypti but with Ae. albopictus present. Moreover, the SCl can be used to investigate the relative contributions of $A$ e. albopictus and $A$ e. aegypti to dengue virus outbreaks in the absence of entomology data in future research. 


\section{Abbreviations}

Aedes: Ae.; SCl: Suitable conditions index; NB: negative binomial; NDVI: Normalized Difference Vegetation Index; BIC: Bayesian information criterion

\section{Declarations}

\section{Acknowledgements}

We are grateful to the Chinese Center for Disease Control and Prevention (CDC) for providing dengue case data in Guangdong. We also thank NASA's Moderate Resolution Imaging Spectroradiometer (MODIS) for providing the Normalised Difference Vegetation Index (NDVI) data and the National Bureau of Statistics of China for providing population census data.

\section{Funding}

This study was supported by the National Health and Medical Research Council, Australia (APP 1138622). The content of this publication is solely the authors' responsibility and does not reflect the views of the Australian National Health and Medical Research Council. The funder had no role in study design, data collection and analysis, decision to publish, or manuscript preparation.

\section{Available data and materials}

Data supporting the conclusions of this article are included within the article. In addition, the datasets used and analysed during the study are available from the corresponding author upon reasonable request.

\section{Authors' contributions}

Conceptualisation: WH; Methodology: WH, HB, FF, XH, GD; Formal analysis: WH, XL, XH; Writing - Original Draft Preparation: XL, Writing - Review \& Editing: XL, WH, HB, FF, XH, CD, GD. All authors read and approved the final manuscript.

\section{Ethics approval and consent to participate}

This study was approved by the University Human Research Ethics Committee of Queensland University of Technology (Ref: 1800000058).

\section{Consent for publication}

Not applicable.

\section{Competing interests}

The authors declare that they have no competing interests.

\section{References}

1. Bhatt S, Gething PW, Brady OJ, Messina JP, Farlow AW, Moyes CL, et al. The global distribution and burden of dengue. Nature. 2013;496(7446):504-7. doi: 10.1038/nature12060.

2. Brady OJ, Gething PW, Bhatt S, Messina JP, Brownstein JS, Hoen AG, et al. Refining the global spatial limits of dengue virus transmission by evidence-based consensus. PLoS Negl Trop Dis. 2012;6(8):e1760. Epub 2012/08/11. doi: 10.1371/journal.pntd.0001760. PubMed PMID: 22880140; PubMed Central PMCID: PMCPMC3413714.

3. World Health Organization. Dengue and severe dengue. Available from: https://www.who.int/news-room/factsheets/detail/dengue-and-severe-dengue.

4. Guzman MG, Harris E. Dengue. The Lancet. 2015;385(9966):453-65. 
5. Guzman MG, Halstead SB, Artsob H, Buchy P, Farrar J, Gubler DJ, et al. Dengue: a continuing global threat. Nature reviews microbiology. 2010;8(12):S7-S16.

6. Simmons CP, Farrar JJ, van Vinh Chau N, Wills B. Dengue. New England Journal of Medicine. 2012;366(15):1423-32.

7. Guzman MG, Gubler DJ, Izquierdo A, Martinez E, Halstead SB. Dengue infection. Nature reviews Disease primers. 2016;2(1):125.

8. Xie H, Zhou H, Yang Y. Advances in the research on the primary dengue vector Aedes aegypti in China. Chinese Journal of Vector Biology and Control. 2011;22(2):194-7 (in Chinese).

9. Dao-fang; G, Hong-ning; Z. Progress in Dengue fever important vector Aedes albopctus in China. Chin J Vector Biol \& Control. 2009;20(6):607-10 (In Chinese).

10. Wu F, Liu QY, Lu L, Wang JF, Song XP, Ren DS. Distribution of Aedes albopictus (Diptera: Culicidae) in Northwestern China. Vector-Borne and Zoonotic Diseases. 2011;11(8):1181-6. doi: 10.1089/vbz.2010.0032. PubMed PMID: WOS:000293629600027.

11. Brady OJ, Hay SI. The global expansion of dengue: how Aedes aegypti mosquitoes enabled the first pandemic arbovirus. Annual review of entomology. 2020;65:191-208.

12. Lambrechts L, Scott TW, Gubler DJ. Consequences of the Expanding Global Distribution of Aedes albopictus for Dengue Virus Transmission. Plos Neglected Tropical Diseases. 2010;4(5). doi: 10.1371/journal.pntd.0000646. PubMed PMID: WOS:000278601000002.

13. Qiu F-X, Chen Q-Q, Ho Q-Y, Chen W-Z, Zhao Z-G, Zhao B-W. The first epidemic of dengue hemorrhagic fever in the People's Republic of China. The American journal of tropical medicine and hygiene. 1991;44(4):364-70.

14. Zhang F-C, Zhao H, Li L-H, Jiang T, Hong W-X, Wang J, et al. Severe dengue outbreak in Yunnan, China, 2013. International journal of infectious diseases. 2014;27:4-6.

15. Wu J-Y, Lun Z-R, James AA, Chen X-G. Dengue fever in mainland China. The American journal of tropical medicine and hygiene. 2010;83(3):664.

16. Peng H-J, Lai H-B, Zhang Q-L, Xu B-Y, Zhang H, Liu W-H, et al. A local outbreak of dengue caused by an imported case in Dongguan China. BMC public health. 2012;12(1):1-8.

17. Cheng Q, Jing Q, Spear RC, Marshall JM, Yang Z, Gong P. Climate and the Timing of Imported Cases as Determinants of the Dengue Outbreak in Guangzhou, 2014: Evidence from a Mathematical Model. PLoS Negl Trop Dis. 2016;10(2):e0004417. Epub 2016/02/11. doi: 10.1371/journal.pntd.0004417. PubMed PMID: 26863623; PubMed Central PMCID: PMCPMC4749339.

18. Dengue: Guidelines for Diagnosis, Treatment, Prevention and Control: New Edition. Geneva: World Health Organization; 2009. Available from: https://www.ncbi.nlm.nih.gov/books/NBK143157/.

19. Chadee D. Dengue cases and Aedes aegypti indices in Trinidad, West Indies. Acta Tropica. 2009;112(2):174-80.

20. Bowman LR, Runge-Ranzinger S, McCall P. Assessing the relationship between vector indices and dengue transmission: a systematic review of the evidence. PLoS neglected tropical diseases. 2014;8(5):e2848.

21. Lin $\mathrm{C}-\mathrm{H}$, Wen $\mathrm{T}-\mathrm{H}$. Using geographically weighted regression (GWR) to explore spatial varying relationships of immature mosquitoes and human densities with the incidence of dengue. International journal of environmental research and public health. 2011;8(7):2798-815.

22. Mordecai EA, Cohen JM, Evans MV, Gudapati P, Johnson LR, Lippi CA, et al. Detecting the impact of temperature on transmission of Zika, dengue, and chikungunya using mechanistic models. PLoS Negl Trop Dis. 2017;11(4):e0005568. Epub 2017/04/28. doi: 10.1371/journal.pntd.0005568. PubMed PMID: 28448507; PubMed Central PMCID: PMCPMC5423694.

23. Barrera R, Amador M, MacKay AJ. Population dynamics of Aedes aegypti and dengue as influenced by weather and human behavior in San Juan, Puerto Rico. PLoS neglected tropical diseases. 2011;5(12):e1378.

24. Campbell KM, Lin C, lamsirithaworn S, Scott TW. The complex relationship between weather and dengue virus transmission in Thailand. The American journal of tropical medicine and hygiene. 2013;89(6):1066.

25. Reiter P. Climate change and mosquito-borne disease. Environmental health perspectives. 2001;109(suppl 1):141-61. 
26. Yang H, Macoris M, Galvani K, Andrighetti M, Wanderley D. Assessing the effects of temperature on the population of Aedes aegypti, the vector of dengue. Epidemiology \& Infection. 2009;137(8):1188-202.

27. Tun-Lin W, Burkot T, Kay B. Effects of temperature and larval diet on development rates and survival of the dengue vector Aedes aegypti in north Queensland, Australia. Medical and veterinary entomology. 2000;14(1):31-7.

28. KAMIMURA K, Matsuse IT, Takahashi H, Komukai J, Fukuda T, Suzuki K, et al. Effect of temperature on the development of Aedes aegypti and Aedes albopictus. Medical entomology and zoology. 2002;53(1):53-8.

29. Couret J, Dotson E, Benedict MQ. Temperature, larval diet, and density effects on development rate and survival of Aedes aegypti (Diptera: Culicidae). PloS one. 2014;9(2):e87468.

30. Mordecai EA, Cohen JM, Evans MV, Gudapati P, Johnson LR, Lippi CA, et al. Detecting the impact of temperature on transmission of Zika, dengue, and chikungunya using mechanistic models. Plos Neglected Tropical Diseases. 2017;11(4). doi: 10.1371/journal.pntd.0005568. PubMed PMID: WOS:000402256700072.

31. Davis C, Murphy AK, Bambrick H, Devine GJ, Frentiu FD, Yakob L, et al. A regional suitable conditions index to forecast the impact of climate change on dengue vectorial capacity. Environmental Research. 2021;195:110849.

32. National Bureau of Statistics of China. Communiqué of the Seventh National Population Census [1] (No. 3) - Population by Region. May 11, 2021 [9/9/2021]. Available from:

http://www.stats.gov.cn/english/PressRelease/202105/t20210510_1817188.html.

33. Liu K, Hou X, Ren Z, Lowe R, Wang Y, Li R, et al. Climate factors and the East Asian summer monsoon may drive large outbreaks of dengue in China. Environmental research. 2020;183:109190.

34. Lai S, Huang Z, Zhou H, Anders KL, Perkins TA, Yin W, et al. The changing epidemiology of dengue in China, 1990-2014: a descriptive analysis of 25 years of nationwide surveillance data. BMC medicine. 2015;13(1):1-12.

35. Ministry of Health of the People's Republic of China. Diagnostic criteria for dengue fever (WS 216-2008) [in Chinese]. Beijing: People's Medical Publishing House; 2008. p. 1-17.

36. Zhengxing W, Chuang L, Alfredo H. From AVHRR-NDVI to MODIS-EVI: Advances in vegetation index research. Acta ecologica sinica. 2003;23(5):979-87.

37. Didan K, Munoz AB, Solano R, Huete A. MODIS vegetation index user's guide (MOD13 series). University of Arizona: Vegetation Index and Phenology Lab. 2015.

38. xtnbreg - Stata. https://www.stata.com/manuals/xtxtnbreg.pdf.

39. Xu L, Stige LC, Chan K-S, Zhou J, Yang J, Sang S, et al. Climate variation drives dengue dynamics. Proceedings of the National Academy of Sciences. 2017;114(1):113-8.

40. Wesolowski A, Qureshi T, Boni MF, Sundsøy PR, Johansson MA, Rasheed SB, et al. Impact of human mobility on the emergence of dengue epidemics in Pakistan. Proceedings of the National Academy of Sciences. 2015;112(38):11887-92.

41. Zhu G, Liu T, Xiao J, Zhang B, Song T, Zhang Y, et al. Effects of human mobility, temperature and mosquito control on the spatiotemporal transmission of dengue. Science of the Total Environment. 2019;651:969-78.

42. Wu P-C, Lay J-G, Guo H-R, Lin C-Y, Lung S-C, Su H-J. Higher temperature and urbanization affect the spatial patterns of dengue fever transmission in subtropical Taiwan. Science of the total Environment. 2009;407(7):2224-33.

43. Li Y, Kamara F, Zhou G, Puthiyakunnon S, Li C, Liu Y, et al. Urbanization increases Aedes albopictus larval habitats and accelerates mosquito development and survivorship. PLoS neglected tropical diseases. 2014;8(11):e3301.

44. Wimberly MC, Davis JK, Evans MV, Hess A, Newberry PM, Solano-Asamoah N, et al. Land cover affects microclimate and temperature suitability for arbovirus transmission in an urban landscape. PLoS neglected tropical diseases.

2020;14(9):e0008614.

45. Barrera R, Amador M, Diaz A, Smith J, Munoz-Jordan J, Rosario Y. Unusual productivity of Aedes aegypti in septic tanks and its implications for dengue control. Medical and veterinary entomology. 2008;22(1):62-9.

46. Thongsripong P, Green A, Kittayapong P, Kapan D, Wilcox B, Bennett S. Mosquito vector diversity across habitats in central Thailand endemic for dengue and other arthropod-borne diseases. PLoS neglected tropical diseases. 2013;7(10):e2507.

47. Endy TP, Nisalak A, Chunsuttiwat S, Libraty DH, Green S, Rothman AL, et al. Spatial and temporal circulation of dengue virus serotypes: a prospective study of primary school children in Kamphaeng Phet, Thailand. American journal of epidemiology.

Page $11 / 14$ 
2002;156(1):52-9.

48. Yang BY, Borgert BA, Alto BW, Boohene CK, Brew J, Deutsch K, et al. Modelling distributions of Aedes aegypti and Aedes albopictus using climate, host density and interspecies competition. Plos Neglected Tropical Diseases. 2021;15(3). doi: 10.1371/journal.pntd.0009063. PubMed PMID: WOS:000634794600008.

49. Tsai P-J, Teng H-J. Role of Aedes aegypti (Linnaeus) and Aedes albopictus (Skuse) in local dengue epidemics in Taiwan. BMC infectious diseases. 2016;16(1):1-20.

50. Chen Zong-jing, Qin Bing, Bai An-ying et. al. An experimental study of interspecific competition between Aedes aegypti from Wushi town of Leizhou and Ae. albopictus from different places in Guangdong province, China. Chin J Vector Biol \& Control, . 2020;31(4):486-9.

51. Liu B, Gao X, Ma J, Jiao Z, Xiao J, Hayat MA, et al. Modeling the present and future distribution of arbovirus vectors Aedes aegypti and Aedes albopictus under climate change scenarios in Mainland China. Science of the Total Environment. 2019;664:203-14.

\section{Figures}

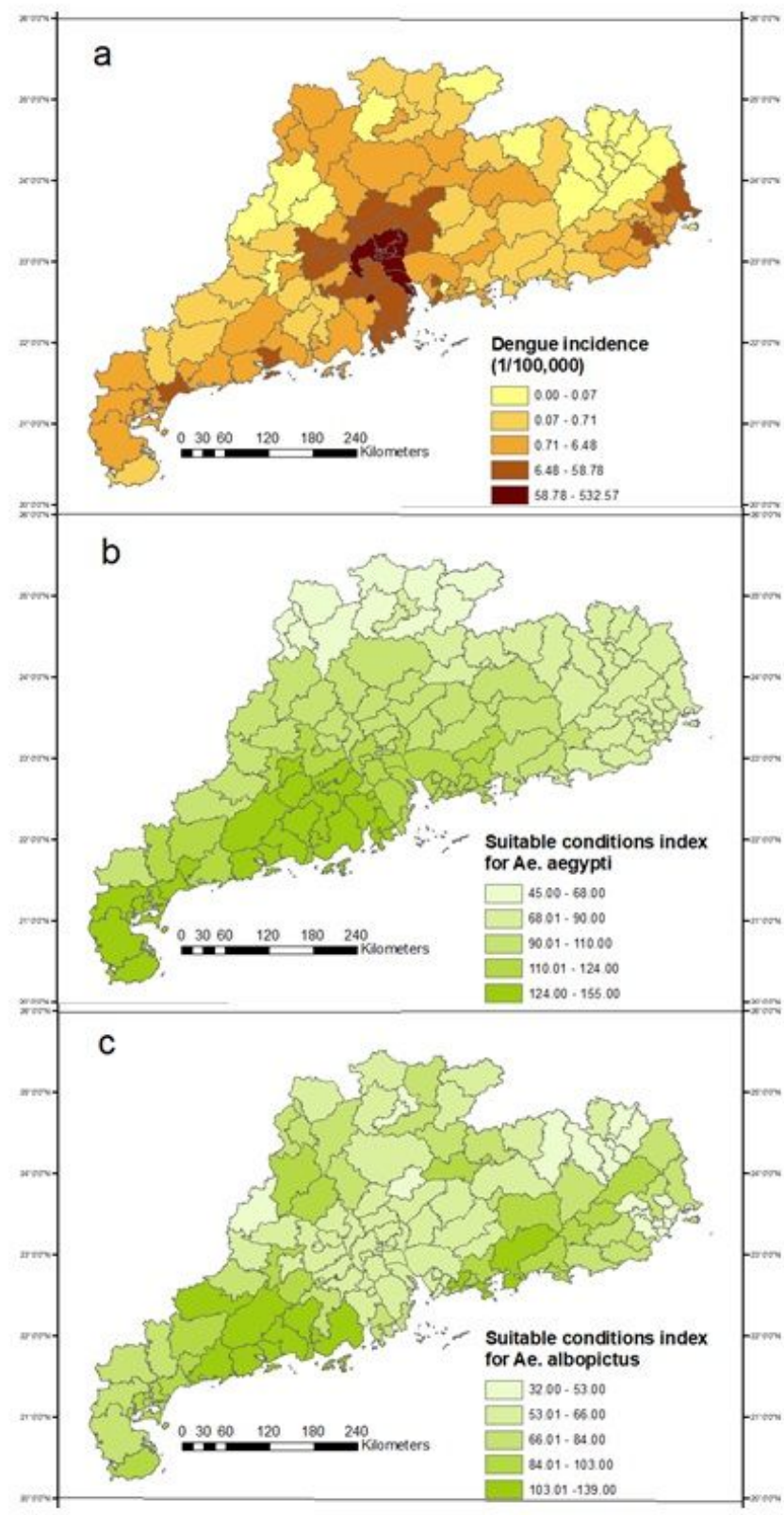


Figure 1

Geographical distribution of dengue (a) and suitable condition index (SCl) for Ae. aegypti (b) and SCl for Ae. albopictus (c) at a county level in Guangdong province, China, 2014
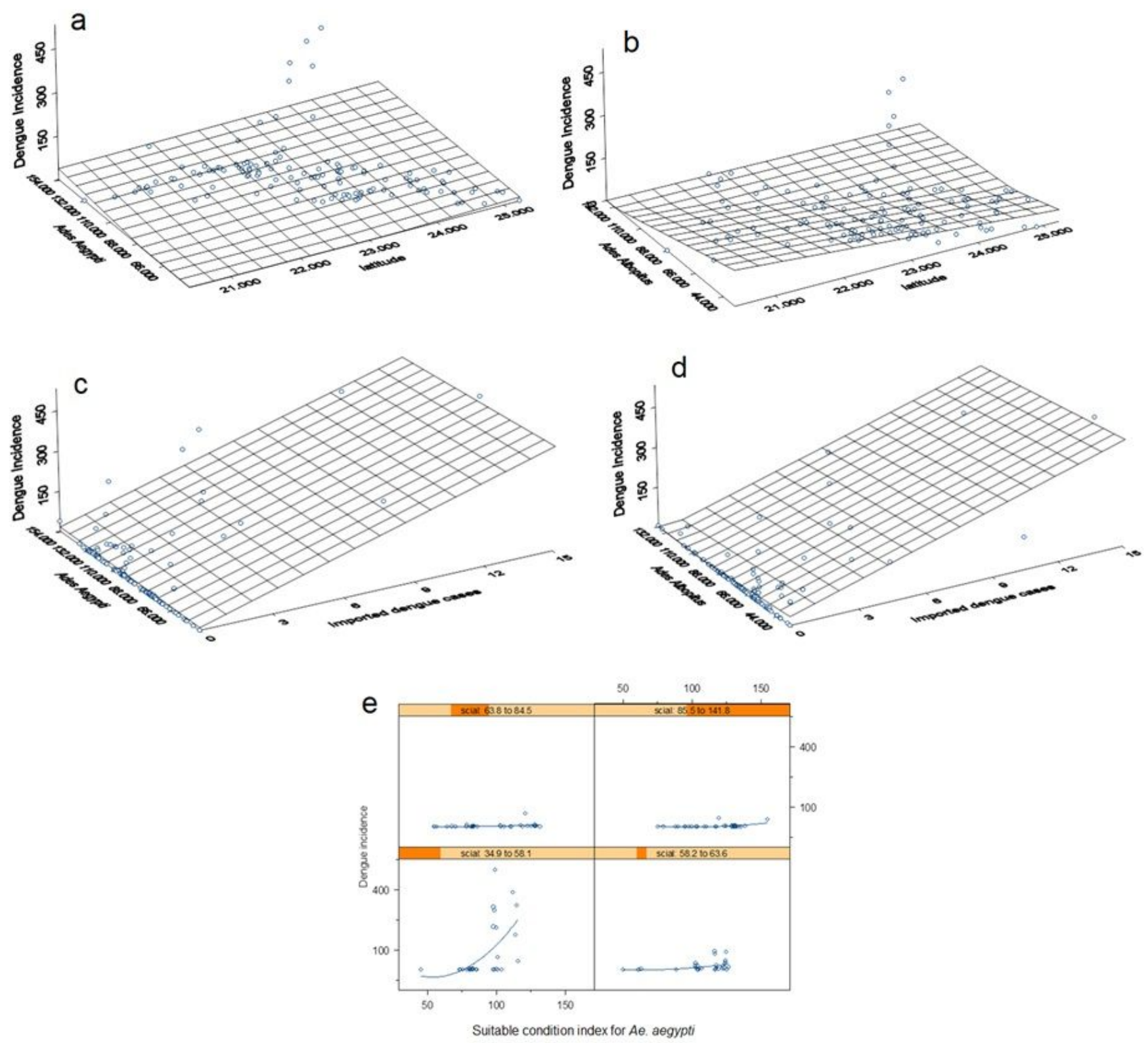

Figure 2

The relationship between autochthonous dengue incidence, latitude, imported dengue cases and suitable conditions index (SCl) in Guangdong, 2014. The relationship between autochthonous dengue incidence, latitude and SCI for Ae. aegypti (a); the relationship between autochthonous dengue incidence, latitude and $\mathrm{SCl}$ for $A$ e. albopictus (b); the relationship between autochthonous dengue incidence and imported dengue cases and SCI for Ae. aegypti (c); the relationship between autochthonous dengue incidence and imported dengue cases and SCI for Ae. albopictus (d) and the relationship between autochthonous dengue incidence and SCI for Ae. aegypti over various levels of SCl for Ae. albopictus ("scial" for short) (e).

\section{Supplementary Files}


This is a list of supplementary files associated with this preprint. Click to download.

- GraphicalAbstract.tif 